14

\title{
Анализ электростатического взаимодействия димерных комплексов. I. Метод подбора ингибиторов к производным белка АРP
}

\author{
(C) Т.В. Кошлан, ${ }^{1}$ К.Г. Куликов ${ }^{2}$ \\ ${ }^{1}$ Санкт-Петербургский государственный университет, \\ 199034 Санкт-Петербург, Россия \\ ${ }^{2}$ Санкт-Петербургский политехнический университет Петра Великого, \\ 195251 Санкт-Петербург, Россия \\ e-mail: kulikov.kirill.g@gmail.com
}

Поступило в Редакцию 27 декабря 2019 г.

В окончательной редакции 27 декабря 2019 г.

Принято к публикации 24 января 2020 г.

\begin{abstract}
Представлен оригинальный метод, позволяющий получить качественную оценку способности амилоидных пептидов образовывать высокомолекулярные структуры, решать проблему стабильности амилоидных димерных комплексов при помощи введенных величин: логарифм числа обусловленности матрицы потенциальной энергии попарного электростатического взаимодействия между аминокислотными остатками и дифференциальной энтропии для случая многомерного нормального распределения, что позволило определить образование структур более высокого порядка: олигомеров, протофибрилл, фибрилл по мере достижения состояния стабильности. Таким образом, основным направлением в блокировании формирования амилоидов при образовании ими высокомолекулярных структур является обеспечение достижения амилоидами стабильного состояния на уровне димерных комплексов.
\end{abstract}

Ключевые слова: болезнь Альцгеймера, амилоиды, ключевые аминокислотные остатки, дифференциальная энтропия, число обусловленности.

DOI: $10.21883 /$ JTF.2020.07.49459.427-19

\section{Введение}

Болезнь Альцгеймера (БА) является прогрессирующим необратимым неврологическим расстройством с выраженной атрофией коры головного мозга и потерей кортикальных и подкорковых нейронов, которая патологически характеризуется скопление амилоидных бляшек и многочисленных нейрофибриллярных клубков [1].

Главными характеристиками БА, позволяющими поставить диагноз под микроскопическим исследованием, являются сенильные бляшки и сети нейрофибрилл. Наблюдается прогрессирующая и впоследствии массовая гибель нейронов и реактивный глиоз в местах образования сенильных бляшек [2].

Сенильные бляшки представляют собой фокальные сферические скопления расширенных и извитых отростков нейронов, расположенных обычно вокруг амилоидного центра. Амилоидный центр содержит патологический белок. Главный компонент амилоидного центра сенильных бляшек - белок $A \beta$, производное более крупной молекулы - АРР. Аминокислотные последовательности двух основных разновидностей белка $A \beta$, обозначаемых $A \beta_{40}$ и $A \beta_{42}$, имеют одинаковый $\mathrm{N}$-концевой участок и отличаются на 2 аминокислотных остатка в С-концевом участке. Другие белки, присутствующие в сенильных бляшках, малочисленны [2].

Перейдем к более подробному рассмотрению амилоидов. Было обнаружено, что $A \beta$-амилоид состоит из более чем 40 аминокислот, связанных вместе, сенильные бляшки представляют собой отвердевшую агрегацию $A \beta$-амилоида.

В 1987 г. группа во главе с Jie Kang et al. в Германии идентифицировала белок-предшественник амилоида (AРP) [3]. Было обнаружено, что АРР присутствует в клетках головного мозга, а $A \beta$-амилоид представляет собой фрагмент АРP, расщепляемый ферментами. Они также обнаружили, что APP был трансмембранным белком. Более того, было обнаружено, что ген АРP находится на 21 хромосоме [3]. Эта идентификация АРP привела к появлению амилоидной гипотезы [4-6].

Патологический процесс может быть объяснен амилоидопатией. $A \beta$ является высоко когезивным белком с молекулярной массой приблизительно 4000 кДа, формирует старческие бляшки и цереброваскулярные амилоиды. АРР представляет собой трансмембранный белок типа I и расщепляется $A$-секретазой, $\beta$-секретазой и $\gamma$-секретазой.

Однако $A \beta$ образуется поэтапным расщеплением $\beta$-секретазой и $\gamma$-секретазой. При этом продуцируется два основных молекулярных вида $A \beta$ : $A \beta_{40}$, который имеет 40 аминокислотных остатков, и высоко когезивный $A \beta_{42}$, который на два аминокислотных остатка длиннее и откладывается на ранней стадии, образует сенильные бляшки, которые являются характерными патологическими изменениями. При этом $A \beta$ либо откладывается в межклеточном пространстве церебральной паренхимы с образованием сенильных бляшек или откладывается на стенке мозга и образует амилоидную ангиопатию [3]. 
Церебральная амилоидная ангиопатия связана с отложением $\beta$-амилоида в мозговых сосудах. Часто встречается в пожилом возрасте, она может вызвать спонтанное внутримозговое кровоизлияние, вносит вклад в развитие когнитивных нарушений, способствует возникновению геморрагических осложнений антитромботической и тромболитической терапий [4].

Разработанный в настоящей работе метод позволит анализировать вклад каждого аминокислотного остатка $A \beta_{(1-40)}$ и $A \beta_{(1-42)}$ в стабильность димерных комплексов и их склонности вступать в биохимические реакции до образования олигомеров, фибрилл, амилоидов.

Предполагается, что образования структур более высокого порядка (олигомеров, протофибрилл, фибрилл, амилоидов) происходит по причине недостаточной стабильности димеров с учетом $A \beta$-пептидов и таким образом наращивание структуры будет происходить до достижения максимального уровня стабильности биохимическим комплекса.

В настоящей работе будет оцениваться стабильность димерных комплексов посредством создания градуировочной шкалы, которая будет характеризовать величину стабильности образованных димеров при участии $A \beta$-пептидов с различными миссенс мутациями.

В качестве критериев, определяющих стабильность димерного комплекса, мы будем использовать:

1. Меру изменения энтропии $(\Delta H)$.

2. Число обусловленности матрицы, элементы которой являются потенциальные энергии электростатического взаимодействия между попарно взятыми аминокислотными остатками белков. В данной физической постановки задачи оно будет характеризовать степень устойчивости конфигурации биологического комплекса. При этом для выбора более устойчивого биохимического соединения между белками мы выбираем матрицу потенциальной энергии электростатического взаимодействия с наименышим значением $\lg (\operatorname{cond}(w))$ [8-10].

Настоящая работа состоит из несколько частей. Первая часть работы посвящена выводу формулы вычисления дифференциальной энтропии для случая нормального распределения и определение ее меры изменения. Во второй части работы представлены результаты численных расчетов. В заключении приведены основные выводы.

\section{1. Дифференциальная энтропия для многомерного нормального распределения}

Предположим, что случайный вектор $\mathbf{Y}=\left(Y_{1}, Y_{2}, \ldots, Y_{m}\right)$ имеет многомерное нормальное распределение с плотностью распределения

$$
f_{\mathbf{Y}}(\mathbf{x})=\frac{1}{(2 \pi)^{\frac{m}{2}}|\mathbf{\Sigma}|^{\frac{1}{2}}} \exp \left[-\frac{1}{2}(\mathbf{x}-\mathbf{a})^{T} \boldsymbol{\Sigma}^{-1}(\mathbf{x}-\mathbf{a})\right],
$$

и ковариационной матрицей

$$
\begin{aligned}
\boldsymbol{\Sigma} & =\left[\sigma_{Y_{i}, Y_{j}}\right]_{m \times m} \\
& =\left(\begin{array}{cccc}
\sigma_{Y_{1}}^{2} & \operatorname{cov}\left(Y_{1}, Y_{2}\right) & \ldots & \operatorname{cov}\left(Y_{1}, Y_{m}\right) \\
\operatorname{cov}\left(Y_{2}, Y_{1}\right) & \sigma_{Y_{2}}^{2} & \ldots & \operatorname{cov}\left(Y_{2}, Y_{m}\right) \\
\vdots & \vdots & \ddots & \vdots \\
\operatorname{cov}\left(Y_{m}, Y_{1}\right) & \operatorname{cov}\left(Y_{m}, Y_{2}\right) & \ldots & \sigma_{Y_{m}}^{2}
\end{array}\right),
\end{aligned}
$$

где $|\boldsymbol{\Sigma}| \quad-\quad$ определитель матрицы $\boldsymbol{\Sigma}$, $a=\left(a_{1}, a_{2}, \ldots, a_{m}\right)^{T} \quad-\quad$ вектор $\quad$ математических ожиданий, $\sigma_{Y_{i}}^{2}$ - дисперсия, $i=\overline{1, m}$.

Теорема. Пусть $\boldsymbol{\Sigma}$ - ковариационная матрица случайного нормально распределенного вектора $\mathbf{Y}$, определенная выражением (2). Тогда энтропия вектора $\mathbf{Y}$ есть

$$
H(\mathbf{Y})=\frac{1}{2} \ln \left[(2 \pi e)^{m}|\mathbf{\Sigma}|\right]
$$

Доказательство данной теоремы приведено в [11].

Вычисление дифференциальной энтропии по формуле (3) представляет собой достаточно сложную вычислительную задачу, так как при ее подсчете необходимо знать ковариации между всеми компонентами, составляющими случайный вектор $\left(Y_{1}, Y_{2}, \ldots, Y_{m}\right)$. Однако существует возможность преодоления указанной трудности с помощью ортогонального преобразования.

Возможность ортогонального преобразования базируется на симметричности и положительной знакоопределенности матрицы $\boldsymbol{\Sigma}$, которое означает, что для любого ненулевого вектора и матричное произведение вида

$$
\mathbf{u} \mathbf{\Sigma} \mathbf{u}^{T} \geq 0 \text {. }
$$

Рассмотрим выражение

$$
\boldsymbol{\Sigma}=\mathbf{M}\left[(\mathbf{Y}-\mathbf{a})^{T}(\mathbf{Y}-\mathbf{a})\right] .
$$

Подставим (5) в (4), тогда получим

$$
\begin{aligned}
& \mathbf{u} \boldsymbol{\Sigma} \mathbf{u}^{T}=\mathbf{u}\left[\mathbf{M}\left[(\mathbf{Y}-\mathbf{a})^{T}(\mathbf{Y}-\mathbf{a})\right]\right] \mathbf{u}^{T} \\
& =\mathbf{M}\left[\mathbf{u}(\mathbf{Y}-\mathbf{a})^{T}(\mathbf{Y}-\mathbf{a}) \mathbf{u}^{T}\right]=\mathbf{M}\left[\left(\mathbf{u}(\mathbf{Y}-\mathbf{a})^{T}\right)^{2}\right] \geq 0,
\end{aligned}
$$

где М - начальный момент первого порядка.

Как известно ортогонализация симметричной и знакоопределенной матрицы означает существование матрицы ортогонального преобразования $\mathbf{Q}$, такой, что матричное произведение $\mathbf{Q} \mathbf{\Sigma} \mathbf{Q}^{T}$ дает диагональную матрицу вида

$$
\Lambda=\left(\begin{array}{ccccc}
\lambda_{1} & \ldots & 0 & \ldots & 0 \\
0 & \ldots & \lambda_{2} & \ldots & 0 \\
\vdots & \vdots & \ddots & \vdots & \\
0 & \ldots & 0 & \ldots & \lambda_{m}
\end{array}\right)
$$

где $\lambda_{i}-$ собственные числа, $i=\overline{1, m}$.

Для определителя матрицы $\boldsymbol{\Lambda}$ справедливо соотношение:

$$
|\boldsymbol{\Lambda}|=\lambda_{1} \lambda_{2} \ldots \lambda_{m}
$$


Перенчень миссенс мутаций и склонность к агрегированию пептида с данными мутациями

\begin{tabular}{|c|c|}
\hline N мутации & Склонность к агрегированию \\
\hline $\mathrm{K} 16 \mathrm{~N}(\mathrm{LYS} 16 \mathrm{ASN}))$ & У гетерозиготных особей - токсичен [14]. У гомозиготных — особей не токсичен \\
\hline A21G(ALA21GLY) & Низкая склонность к агрегированию [15-17] \\
\hline E22G(GLU22GLY) & Образуют фибриллы с повышенной скоростью [18] \\
\hline E22K(GLU22LYS) & Усиленная агрегация олигомеров $[19,20]$ \\
\hline $\begin{array}{l}\text { E22Q(GLU22GLN }) \\
\text { E22G(GLU22GLY })\end{array}$ & $\begin{array}{l}\text { Обширный амилоид L34V(LEU34VAL)) демонстрировал лаг-фазу, сравнимую с фазой wt- } A \beta_{40}, \\
\text { демонстрирующей увеличение сложных структур только после трехдневной инкубации при } 37^{\circ} \mathrm{C} \\
\text { и физиологических концентрациях соли. Однако образование олигомеров высокого массового порядка, } \\
\text { по-видимому, происходит немного быстрее, чем для пептида wt, достигая промежуточных уровней между } \\
\text { A } \beta_{40} \text { и E22Q(GLU22GLN). После } 24 \text { h инкубации при физиологической концентрации соли } \\
\text { wt- } A \beta_{40} \text { и L34V(LEU34VAL) в основном проявляли небольшие глобулярные структуры, которые обычно } \\
\text { предшествуют образованию протофибрилл, тогда как E22Q(GLU22GLN) демонстрировал обильные } \\
\text { протофибриллярные компоненты [21] }\end{array}$ \\
\hline G38S(GLY38SER) & Нет амилоида $[22,23]$ \\
\hline
\end{tabular}

Таким образом, ортогональное преобразование исходной матрицы $\boldsymbol{\Sigma}$ позволяет значительно снизить вычислительную сложность задачи.

Для нахождения собственных чисел матрицы $\boldsymbol{\Sigma}$ может использоваться SVD (сингулярное) разложение.

Определим меру изменения энтропии $(\Delta H)$ для рассматриваемых пептидов с мутациями и пептида дикого типа с учетом выражения (3) изменение энтропии будет иметь вид

$$
\begin{aligned}
\Delta H & =\frac{1}{2} \ln \left[(2 \pi e)^{m}|\boldsymbol{\Sigma}|_{m}\right]-\frac{1}{2} \ln \left[(2 \pi e)^{n}|\boldsymbol{\Sigma}|_{n}\right] \\
& =\ln \left[\frac{(2 \pi e)^{m}|\boldsymbol{\Sigma}|_{m}}{(2 \pi e)^{n}|\boldsymbol{\Sigma}|_{n}}\right],
\end{aligned}
$$

где $|\boldsymbol{\Sigma}|_{m}-$ определитель ковариационной матрицы $\boldsymbol{\Sigma}_{m \times m}$ (пептидов с мутациями) $m$-го порядка, которая определена выражением (2), и $|\boldsymbol{\Sigma}|_{n}$ - определитель ковариационной матрицы $\boldsymbol{\Sigma}_{n \times n}$ (пептида дикого типа) $n$-го порядка, которая также определена выражением (2).

Отметим, что энтропия - это мера неупорядоченности молекулярной системы. При этом беспорядочным состояниям отвечает большое значение энтропии, а упорядоченным - малое значение энтропии.

Таким образом, если процесс идет в направлении увеличения неупорядоченности системы, то величина $\Delta H>0$, определенная выражением (6), показывает, что степень упорядоченности уменьшается относительно пептида дикого типа, если же $\Delta H<0$, то в этом случае степень упорядоченности увеличивается относительно пептида дикого типа.

\section{2. Результаты численных расчетов}

В разд. 2 будут приведены численные результаты, полученные в ходе выполнения и обоснования новой

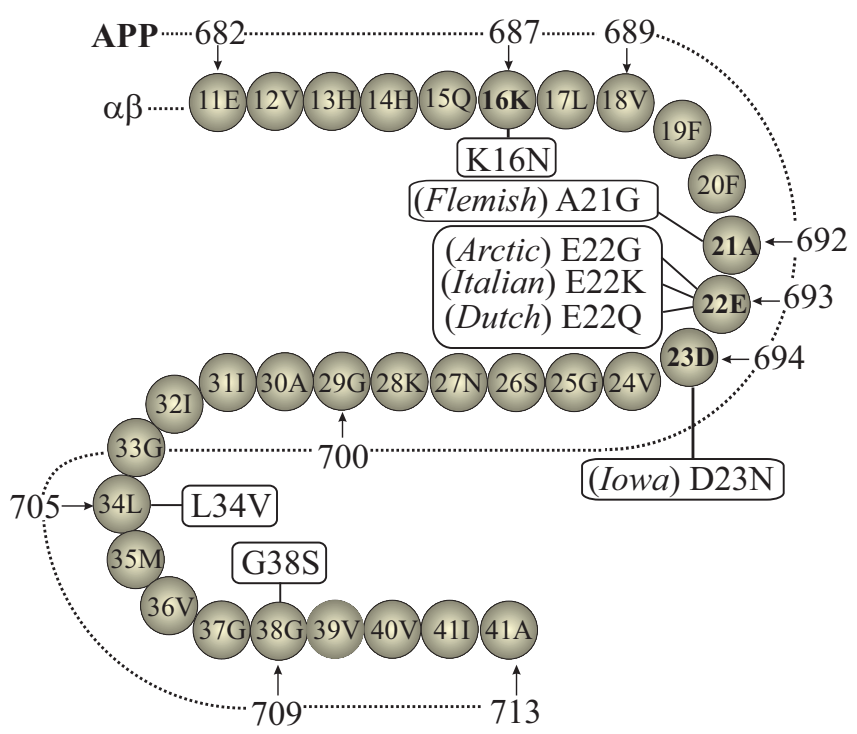

Рис. 1. Аминокислотная последовательность $A \beta_{(11-42)}$ пептида с указанием миссенс мутаций. Также приведена нумерация а.а. пептида, а также нумерация а.а. относительно белка предшественника АРP.

биофизической концепции образования амилоидными пептидами структур более высокого порядка (олигомеров, протофибрилл, фибрилл, амилоидов).

Поскольку представленный в настоящей работе метод касается нового понимания в устойчивости пептидных амилоидов, приведем участок такого амилоидного пептида $A \beta_{(11-42)}$, который отвечает длине аминокислотной последовательности трехмерной структуры из базы PDB:2MXU [5]. На рис. 1 приведена аминокислотная последовательность $A \beta_{(11-42)}$-пептида с указанием миссенс мутаций (см. таблицу), которые влекут за собой различные биологические и патофизиологические эффекты в человеческом организме [23]. 


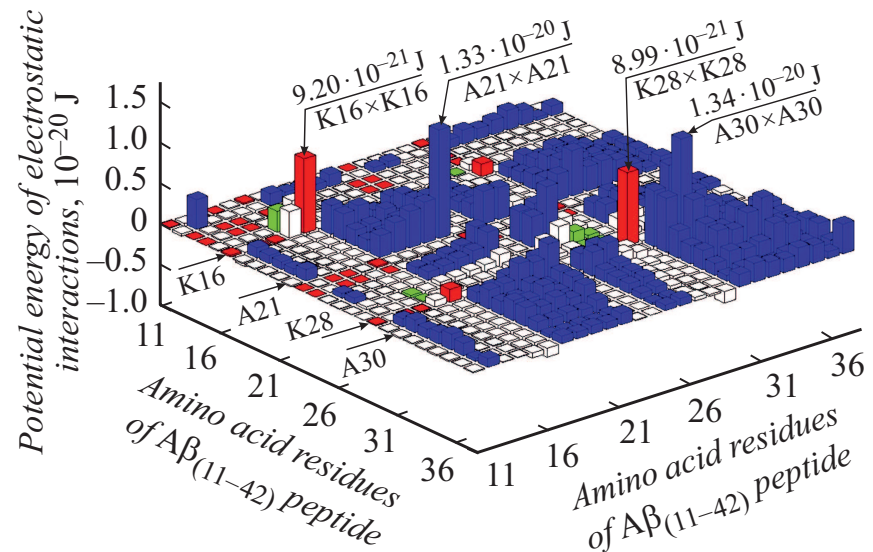

Pис. 2. Трехмерная карта потенциальной энергии электростатического взаимодействия $\left[{ }_{w t} A \beta\right]_{2}$ с указанием максимальных положительных значений.

Авторы статьи воспользовались известной трехмерной структурой из базы PDB: $2 \mathrm{MXU}$ [12]. Из всего множества приведенных в ней пептидов было оставлено только две полипептидные цепи амилоидных пептидов. Далее была рассчитана потенциальная энергия электростатического взаимодействия между аминокислотными остатками двух амилоидных пептидов с учетом трехмерной структуры, и получена матрица потенциальной энергии электростатического взаимодействия между двумя амилоидными пептидами ${ }_{w t} A \beta_{(11-42)}$, построена трехмерная карта потенциальной энергии электростатического взаимодействия (рис. 2). При этом область $C$ конца с учетом аминокислотных остатков I41(ILE41) и A42(ALA42) будет рассмотрена отдельно.

\section{3. Описание карт потенциальной энергии электростатического взаимодействия}

На представленном рис. 2 видно в основном положительную область значений потенциальной энергии электростатического взаимодействия двух одинаковых амилоидных пептидов ${ }_{w t} A \beta_{(11-42)}$, трехмерная структура которых бралась из PDB-базы [12].

Стрелками на рисунке обозначены максимальные значения потенциальной энергии электростатического взаимодействия амилоидных пептидов в положительной области. Эти максимальные значения в основном приходятся на диагональные элементы матрицы: K16(LYS16) со стороны первого амилоидного пептида и K16(LYS16) со стороны второго амилоидного пептида и соответственно A21(ALA21) со стороны I-го ${ }_{w t} A \beta_{(11-42)}$ и A21(ALA21) со стороны II-го ${ }_{w t} A \beta_{(11-42)}$ пептида, K28(LYS28) со стороны I-го ${ }_{w t} A \beta_{(11-42)}$ и K28(LYS28) со стороны II-го ${ }_{w t} A \beta_{(11-42)}$ пептида, A30(ALA30) со стороны I-го ${ }_{w t} A \beta_{(11-42)}$ и A30(ALA30) со стороны II-го ${ }_{w t} A \beta_{(11-42)}$ пептида.
Взаимодействия A42(ALA42) со стороны I-го ${ }_{w t} A \beta_{(11-42)}$ и A42(ALA42) со стороны II-го ${ }_{w t} A \beta_{(11-42)}$ пептида будут рассмотрены отдельно, поскольку физические параметры взаимодействия двух ${ }_{w t} A \beta_{(11-42)}$ имеют некоторые отличия от физических параметров взаимодействия двух ${ }_{w t} A \beta_{(11-40)}$ за счет наличия двух последних аминокислотных остатков I41(ILE41) и A42(ALA42) со стороны $C$-конца амилоидных пептидов [13].

Все дальнейшие расчеты, которые будут касаться взаимодействия амилоидных пептидов, содержащие с 11го по 42-й аминокислотный остаток (а.а.) и будут обозначаться „, $A \beta^{\text {“ } ~ с ~ у к а з а н и е м ~ м у т а ц и и ~ „ m u t “ ~ и ~ п о р я д к о в о г о ~}$ номера а.а. (см. таблицу) или с указанием дикой формы амилоидного пептида „wt“", в котором отсутствуют замены и мутации аминокислотных остатков. Перечень мутаций приведен в таблице.

Перейдем к рассмотрению графика взаимодействия двух мутантных форм амилоидных пептидов тит $A \beta_{(K 16 N)}$

На рис. 3 представлено трехмерное преставление потенциальной энергии при взаимодействии двух ${ }_{m u t} A \beta_{(K 16 N)}$. Стрелкой указан бар, отвечающий мутациям в двух амилоидных пептидах и соответствующая им энергия взаимодействия.

При взаимодействии амилоидных пептидов дикой формы взаимодействие K16(LYS16) I-го ${ }_{w t} A \beta$ и К16(LYS16) II-го ${ }_{w t} A \beta$-пептида соответствовало величине потенциальной энергии $9.2 \cdot 10^{-21} \mathrm{~J}$. При замере K16(LYS16) на N16(ASN16) происходит существенное снижение величины потенциальной энергии взаимодействия до $2.19 \cdot 10^{-22}$ J. График изменения величины потенциальной энергии при взаимодействии K16N(LYS16ASN) со всеми остальными а.а. второго пептида приведен на рис. 4.

Рассмотрим результаты взаимодействия двух мутантных форм амилоидных белков ${ }_{m u t} A \beta_{(A 21 G)}$. Трехмерная

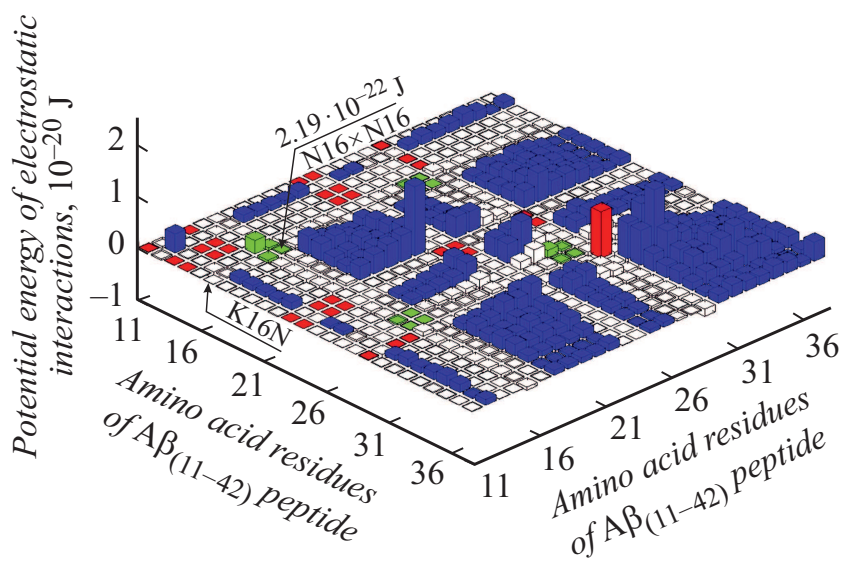

Рис. 3. Трехмерная карта потенциальной энергии электростатического взаимодействия двух мутантных форм тиt $A \beta_{(K 16 N)}$. На карте указан столбец, отвечающий величине взаимодействия двух а.а. мутантных пептидов N16(ASN16) и N16(ASN16). 


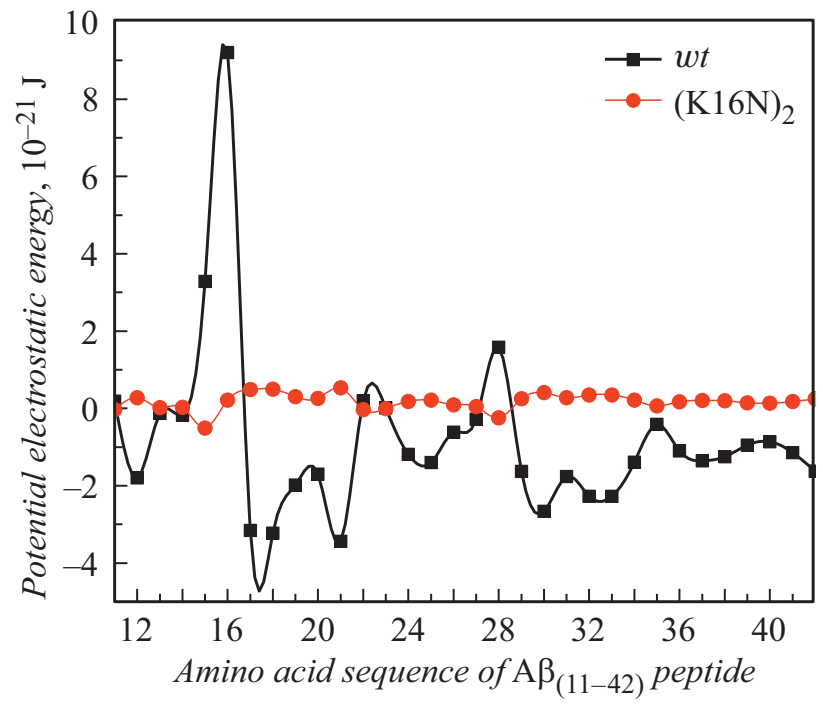

Рис. 4. Кривые потенциальной энергии электростатического взаимодействия а.a. K16(LYS16) со всеми остальными а.а. второго пептида ${ }_{w} A \beta$ (квадратики) и потенциальная энергия попарного электростатического взаимодействия между a.a. N16(ASN16) со всеми остальными а.а. второго пептида ${ }_{m u t} A \beta_{(K 16 N)}$ (кружечки).

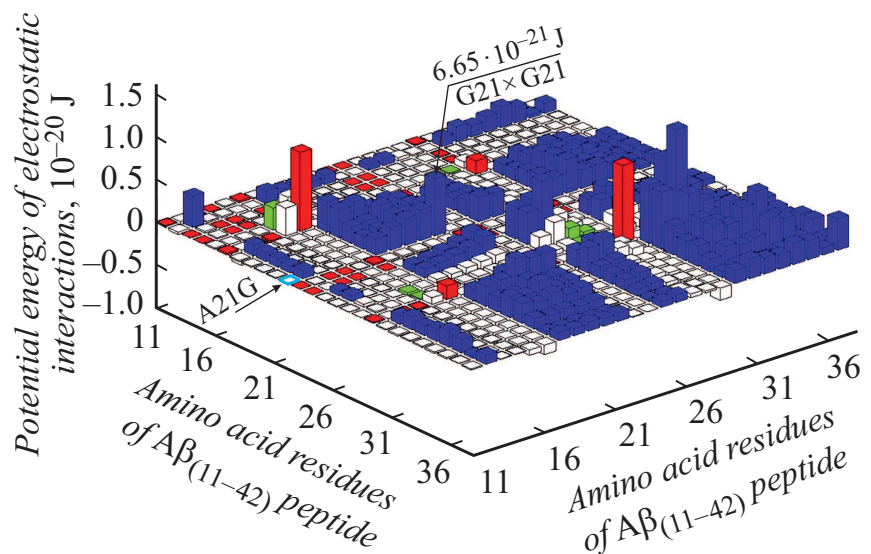

Рис. 5. Трехмерная карта величины потенциальной энергии электростатического взаимодействия двух мутантных форм ${ }_{m u t} A \beta_{(A 21 G)}$. На карте обозначен столбец, отвечающий величине взаимодействия двух а.а. G21(GLY21) и G21(GLY21).

карта потенциальной энергии электростатического взаимодействия представлена на рис. 5.

При взаимодействии амилоидных пептидов дикой формы, взаимодействие A21(ALA21) I-го ${ }_{w t} A \beta$ и A21(ALA21) II-го ${ }_{w t} A \beta$-пептида соответствовало величине потенциальной энергии $1.33 \cdot 10^{-20} \mathrm{~J}$. При замере A21(ALA21) на G21(GLY21) происходит существенное снижение величины потенциальной энергии взаимодействия до $6.65 \cdot 10^{-21} \mathrm{~J}$. График изменения величины потенциальной энергии взаимодействия A21G(ALA21GLY) со всеми остальными а.а. второго пептида приведен на рис. 6.
Для получения более наглядного представления об изменении величины потенциальной энергии при миссенс мутациях в амилоидных пептидах, приведем кривые изменения потенциальной энергии взаимодействия а.а. пептида ${ }_{w t} A \beta$ и а.а. пептида ${ }_{m u t} A \beta$, указанного в таблице, при взаимодействии с остальными а.а. таких же амилоидных пептидов (рис. 4).

На рис. 4 приведены кривые потенциальной энергии при взаимодействии K16(LYS16) (а.а. пептида дикого типа) и N16(ASN16) (а.а. пептида с миссенс мутацией) со всеми остальными а.а. полипептидной цепи.

Кривая взаимодействия $\left[{ }_{w t} A \beta\right]_{2}$ характеризуется гораздо большими флуктуациями значений потенциальной энергии электростатического взаимодействия по сравнению с кривой $\left.{ }_{[m u} A \beta_{(K 16 N)}\right]_{2}$. Таким образом, при замене K16(LYS16) на N16(ASN16) происходит существенное уменьшение значений потенциальной энергии.

На рис. 6 приведены кривые потенциальной энергии электростатического взаимодействия A21(ALA21) и G21(GLY21) со всеми остальными а.а. полипептидной цепи второго пептида при образовании $\left.{ }_{m u t} A \beta_{(A 21 G)}\right]_{2}$ (кружечки) и при образовании $\left[{ }_{w t} A \beta\right]_{2}$ (квадратики). Данные кривые практически повторяют друг друга, кривая взаимодействия пептидов дикого типа имеет несколько большие значения энергии по сравнению с кривой взаимодействия двух мутантных форм.

На рис. 7 приведены кривые изменения потенциальной энергии при взаимодействии E22(GLU22) и G22(GLY22) c a.a. полипептидной цепи второго пептида при образовании $\left[{ }_{m u t} A \beta_{(E 22 G)}\right]_{2}$ (кружечки) и при образовании $\left[{ }_{w t} A \beta\right]_{2}$ (квадратики). При этом кривая взаимодействия мутантных пептидов демонстрирует гораздо больший

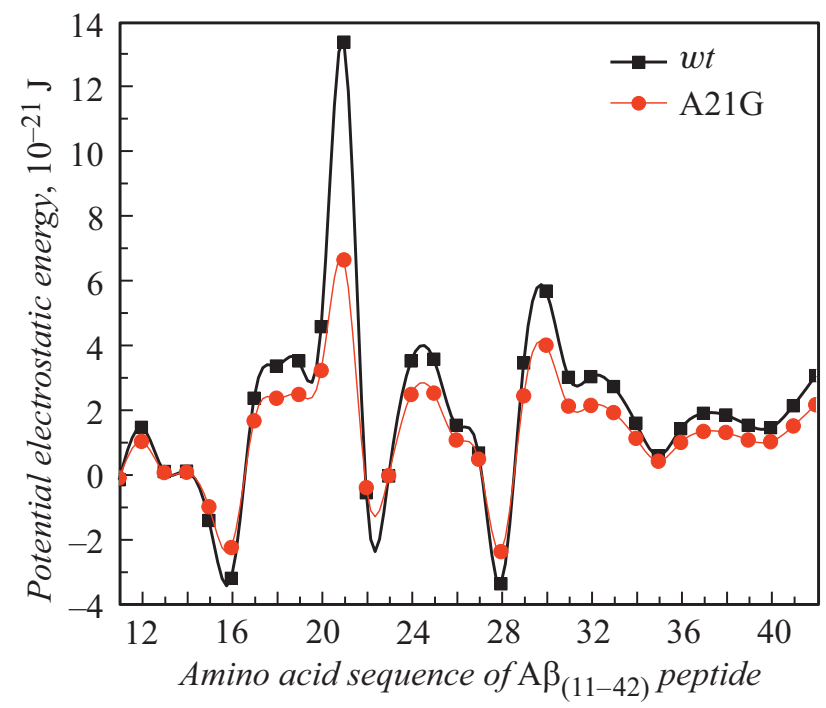

Рис. 6. Кривые потенциальной энергии электростатического взаимодействия а.а. A21(ALA21) со всеми остальными а.а. второго пептида $w_{t} A \beta$ (квадратики) и потенциальная энергия попарного электростатического взаимодействия между а.а. G21(GLY21) со всеми остальными а.а. второго пептида ${ }_{m u t} A \beta_{(A 21 G)}$ (кружечки). 


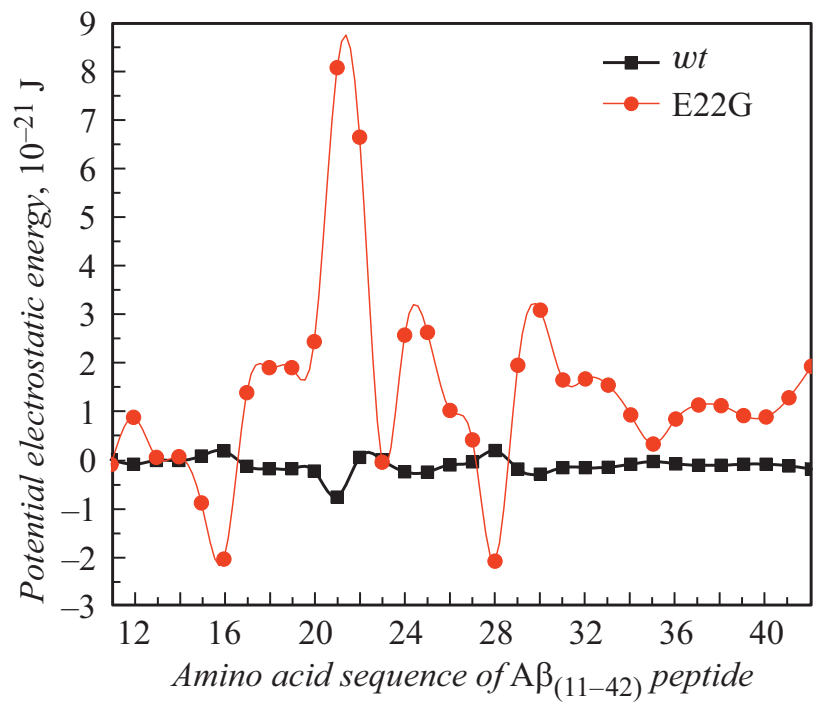

Рис. 7. Кривые потенциальной энергии электростатического взаимодействия а.а. Е22(GLU22) со всеми остальными а.а. второго пептида ${ }_{w t} A \beta$ (квадратики) и потенциальная энергия попарного электростатического взаимодействия между а.а. G22(GLY22) со всеми остальными а.а. второго пептида ${ }_{\text {mut }} A \beta_{(E 22 G)}$ (кружечки).

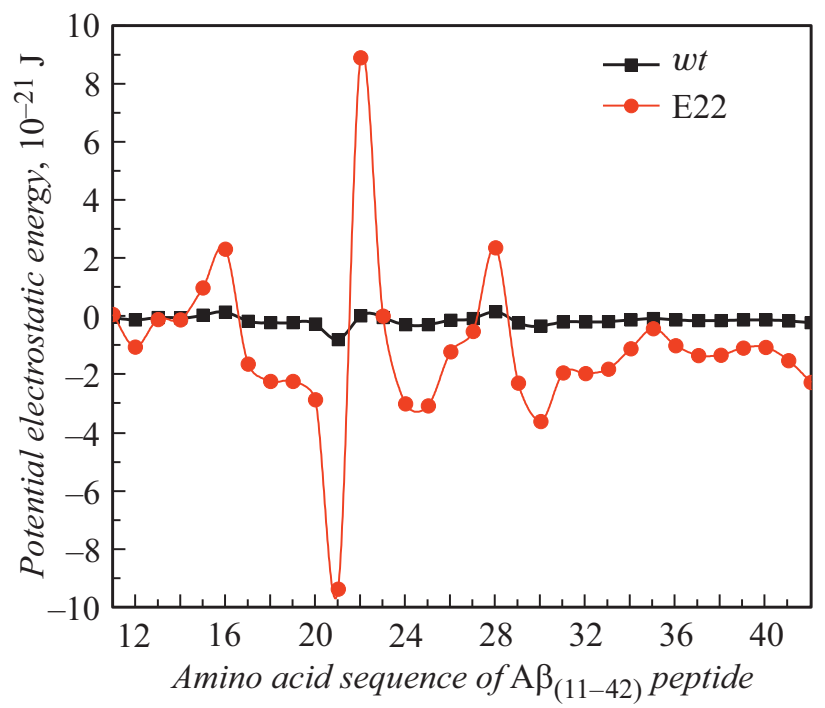

Рис. 8. Кривые потенциальной энергии электростатического взаимодействия а.а. E22(GLU22) со всеми остальными а.а. второго пептида ${ }_{w} A \beta$ (квадратики) и потенциальная энергия попарного электростатического взаимодействия между a.a. K22(LYS22) со всеми остальными а.а. второго пептида ${ }_{\text {mut }} A \beta_{(\text {Е22K) }}$ (кружечки).

разброс значений, чем кривая, полученная для кривых взаимодействия пептидов дикого типа.

На рис. 8 приведены кривые изменения потенциальной энергии при взаимодействии E22(GLU22) и K22(LYS22) c a.a. полипептидной цепи второго пептида при образовании $\left[{ }_{w t} A \beta_{(E 22 K)}\right]_{2}$ (квадратики) и при образовании $\left[{ }_{\text {mut }} A \beta_{(E 22 K)}\right]_{2}$ (кружочки).
Для численной оценки изменения диапазона потенциальной энергии электростатического взаимодействия воспользуемся понятием разности дифференциальной энтропии между взаимодействующими мутантными формами пептидов и взаимодействующими пептидами дикого типа (см. выражение (6)). При этом величина изменения энтропии будет определяться между двумя димерами.

На рис. 9 приведены значения разности дифференциальной энтропии между взаимодействиями мутантных форм амилоидных пептидов и взаимодействием амилоидных пептидов дикого типа.

Анализ расчетных данных позволяет сделать вывод, что миссенс мутации в двух взаимодействующих пептидах, в случаях E22K(GLU22LYS), E22G(GLU22GLY), K16N(LYS16ASN) приводит к тому, что степень упорядоченности уменьшается. Миссенс мутации в двух амилоидных пептидах, в случаях A21G(ALA21GLY), G38S(GLY38SER), наоборот, способствует упорядочиванию системы димера.

Также был проведен анализ стабильности димерных комплексов с учетом пептидов $A \beta$ дикого типа и с учетом пептидов с миссенс мутациями.

Ранее была получена матрица потенциальной энергии между амилоидными пептидами без мутаций и с учетом мутаций, приведенных в таблице.

Графическое представление величины устойчивости биологического комплекса приведено на рис. 10. При этом на графиках указаны наименования мутаций и

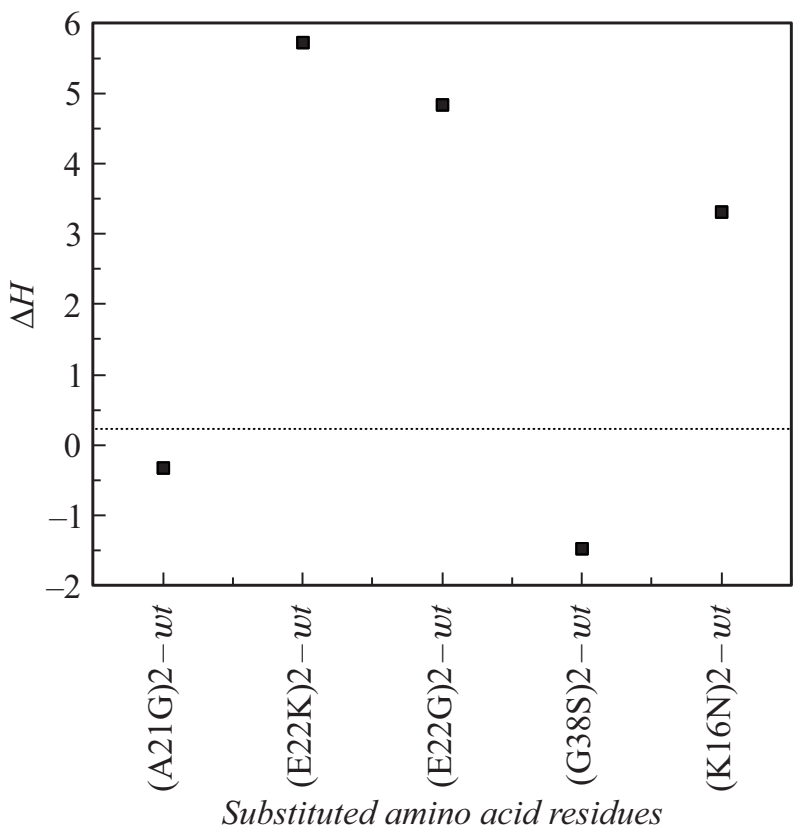

Рис. 9. График изменения энтропии при переходе системы от взаимодействия мутантных форм к взаимодействию диких форм амилоидных пептидов. При этом величина изменения энтропии $(\Delta H)$ безразмерная. Горизонтальная линия на графике определяет границу измения энтропии: область отрицательных значений от области положительных значений. 


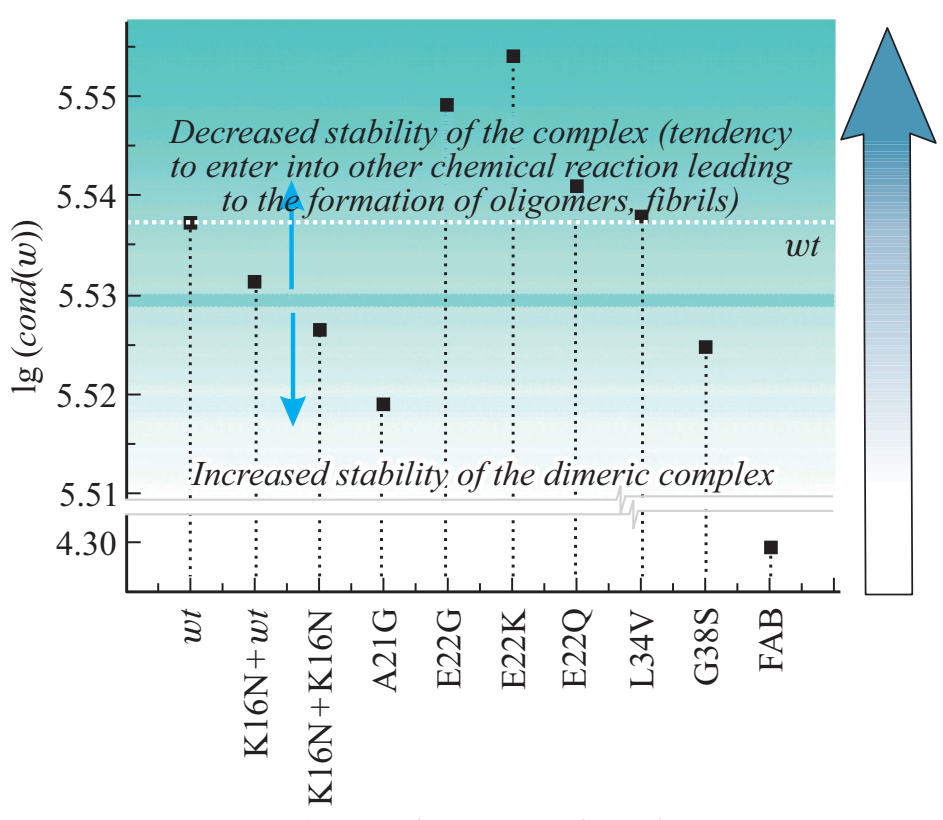

Substituted amino acid residues

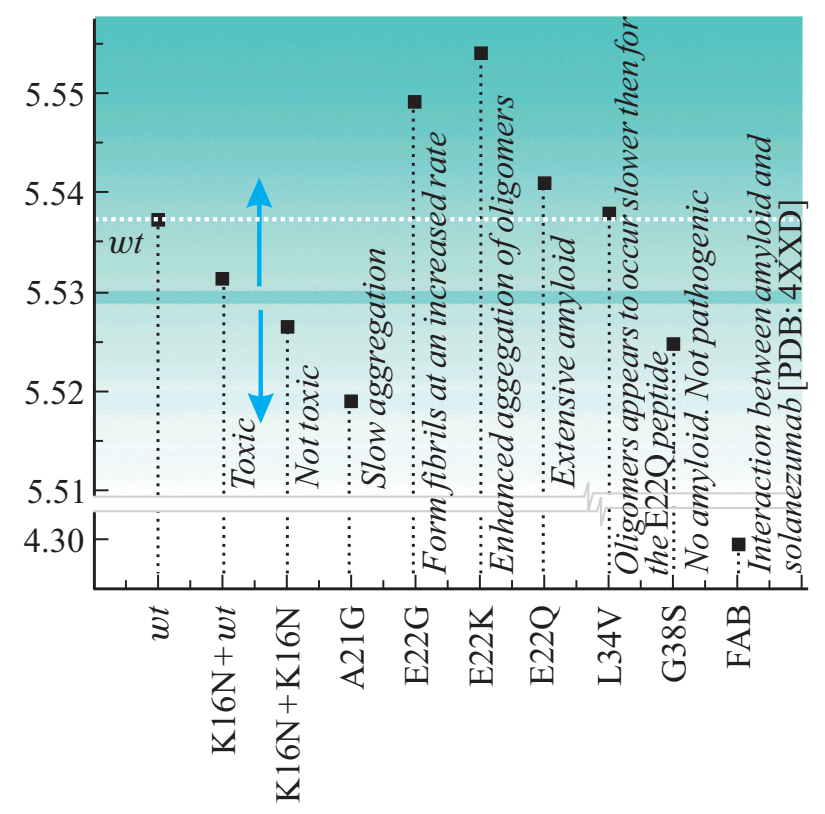

Substituted amino acid residues

Рис. 10. График численных значений критерия стабильности $(\lg (\operatorname{cond}(w)))$, полученных при взаимодействии диких форм ${ }_{w} A \beta$ и при взаимодействии мутантных форм пептидов тин $A \beta$ из таблицы.

характер образования структур более высокого порядка. Большой стрелкой между графиками указано направление значений, характерных для образования более высокомолекулярных форм: олигомеры, протофибриллы, фибриллы. На уровне 5.53 проведена толстая черта, которая условно отделяет структуры, склонные образовывать более высокомолекулярные формы (область выше черты), и область значений, которые характеризуют пониженную склонность к образования молекулярно „тяжелых“ структур по причине увеличения устойчивости димерных комплексов (область ниже черты). Обозначение на графике „FАВ“ отвечает величине стабильности при взаимодействии участка соланезумаба (solanezumab) с амилоидным пептидом, структура в базе PDB : 4XXD [12].

На основании экспериментальных данных о миссенс мутациях и их биологическом эффекте, мы ввели разделительную черту на уровне 5.53 , которая будет отделять мутации в пептидах, ведущие к усиленному формированию структур более высокого порядка от мутаций в пептидах $A \beta$, обладающие пониженной способностью к образованию высокомолекулярных структур. Двумя пороговыми значениями при выборе величины 5.53 являлись взаимодейтсвия пептидов дикого типа $A \beta$, значение величины которых $\lg (\operatorname{cond}(w))$ составило 5.5372 и взаимодейтсвие двух мутантных форм пептида $A \beta$ $\mathrm{K} 16 \mathrm{~N}(\mathrm{LYS16ASN})$, димерная форма которых не влечет за собой токсичных последствий для организма человека. В этом случае значение величины $\lg (\operatorname{cond}(w))$ составило 5.5264. Пунктирной линией на графиках обозначено значение, полученное при взаимодействии пептидов $\left[{ }_{w t} A \beta\right]_{2}$. Две вертикальные стрелки от линии на уровне 5.53 указывают направление в области значений, характеризуемых более низкой степенью стабильности димерных комплексов (стрелка вверх) и более высокой степенью стабильности димерных комплексов (стрелка вниз). Данная градация значений получена и апробирована для трехмерного комплекса из базы PDB:2MXU [12].

Для других трехмерных структур, таких как $5 \mathrm{KKX}$, сохраняется характер распределения димеров $A \beta$ по уровням устойчивости.

\section{Выводы}

В настоящей работе был впервые предложен новый метод, дающий объяснение формированию амилоидных высокомолекулярных структур с точки зрения устойчивости амилоидных комплексов, была приведена численная градация для трехмерной структуры из PDB-базы по степени устойчивости амилоидных димеров. Была введена величина со значением равным 5.53, определяющая устойчивость биокомплекса. При этом она условно разделяла димеры с пониженной и повышенной склонностью образовывать высокомолекулярные структуры.

Величина 5.53 была найдена на основании сопоставления с экспериментальными данными по мутациям в амилоидных пептидах, их физиологических и биофизических характеристиках, способности выступать во все новые биохимические реакции.

Были получены потенциальные энергии электростатического взаимодействия между полипептидными цепями амилоидных пептидов $A \beta_{(11-42)}$, построены трехмерные карты распределения потенциальной энергии, выявлены пиковые значения в положительной области значений. 
Так же следует отметить, что разработанный в работе метод дает хорошее приближение, если замены а.а. не вносят существенных изменений в структуру комплекса. Таким образом, в работе предложен новый взгляд на проблему формирования амилоидов, что позволит поновому подойти к проблеме антиамилоидной терапии, основанной на понимании стабильности амилоидных комплексов.

\section{Конфликт интересов}

Авторы заявляют, что у них нет конфликта интересов.

\section{Список литературы}

[1] Nasica-Labouze J., Nguyen P.H., Sterpone F., Berthoumieu O., Buchete N.V., Cotŭ S., De Simone A., Doig A.J., Faller P., Garcia A., Laio A., Li M.S., Melchionna S., Mousseau $N ., \quad M u$ Y., Paravastu A., Pasquali S., Rosenman D., Strodel B., Tarus B., Viles J.H., Zhang T., Wang C., Derreumaux P. // Chem. Rev. 2015. Vol. 115. N 9. P. 3518-3563.

[2] Кумар Винай, Аббас Абуль К., Нельсон Фаусто, Джсон К. Астер. Основы патологии заболеваний по Роббинсу и Котрану в 3-х томах. Т. 3. М.: Логосфера, 2016. $500 \mathrm{c}$.

[3] Kang J., Lemaire H.G., Unterbeck A., Salbaum J.M., Masters C.L., Grzeschik K.H., Multhaup G., Beyreuther K., Muller-Hill B. // Nature. 1987. Vol. 325. N 6106. P. 733-736.

[4] Glenner G.G., Wong C.W. // Biochem. Biophys. Res. Commun. 1984. Vol. 120. N 3. P. 885-890.

[5] Hardy J., Allsop D. // Trends. Pharmacol. Sci. 1991. Vol. 12. N 10. P. $383-388$.

[6] Selkoe D., Hardy J. // EMBO Mol. Med. 2016. Vol. 8. N 6. P. 595-608.

[7] Кулеш А.А., Дробаха В.Е., Шестаков В.В. // Неврология, нейропсихиатрия, психосоматика. 2018. Т. 10. № 3. C. 4-11.

[8] Koshlan T.V., Kulikov K.G. Mathematical Modeling of Protein Complexes. Springer-Nature, 2018. 367 p.

[9] Кошлан Т.В., Куликов К.Г. // ЖТФ. 2018. Т. 88. Вып. 8. C. 1137-1149.

[10] Кошлан Т.В., Куликов К.Г. // ЖТФ. 2018. Т. 88. Вып. 8. C. $1150-1159$.

[11] Тырсин А.Н., Соколова И.С. // Мат. модел. 2012. Т. 24. № 1. C. 88-102.

[12] Protein data bank-URL:https://www.rcsb.org/(дата обращения 6.12.2019).

[13] Tian Qiu, Qian Liu, Yong-Xiang Chen, Yu-Fen Zhao, YanMei Li // Special Issue: 13th Chinese International Peptide Symposium. 2015. Vol. 21. N 7. P. 522-529.

[14] Kaden D., Harmeier A., Weise C., Munter L.M., Althoff V., Rost B.R., Hildebrand P.W., Schmitz D., Schaefer M., Lurz R., Skodda S., Yamamoto R., Arlt S., Finckh U., Multhaup G. // EMBO Mol. Med. 2012. Vol. 4. N 7. P. 647-659.

[15] Murakami K., Irie K., Morimoto A., Ohigashi H., Shindo M., Nagao M., Shimizu T., Shirasawa T. // Biochem. Biophys. Res. Commun. 2002. Vol. 294. N 1. P. 5-10.
[16] Meinhardt J., Tartaglia G.G., Pawar A., Christopeit T., Hortschansky P., Schroeckh V., Dobson C.M., Vendruscolo M., Fandrich M. // Protein Sci. 2007. Vol. 16. N 6. P. 1214-1222.

[17] Huet A., Derreumaux P. // Biophys. J. 2006. Vol. 91. N 10. P. 3829-3840.

[18] Nilsberth C., Westlind-Danielsson A., Eckman C.B., Condron M.M., Axelman K., Forsell C., Stenh C., Luthman J., Teplow D.B., Younkin S.G., Naslund J., Lannfelt L. // Nat. Neurosci. 2001. Vol. 4. N 9. P. 887-893.

[19] Owen M.C., Gnutt D., Gao M., Wdrmldnder S.K.T.S., Jarvet J., Grdslund A., Winter R., Ebbinghaus S., Strodel B. // Chem. Soc. Rev. 2019. Vol. 48. N 14. P. 3946-3996.

[20] Yang X., Meisl G., Frohm B., Thulin E., Knowles T.P.J., Linse S. // Proc. Natl. Acad. Sci. USA. 2018. Vol. 115. N 26. P. E5849-E5858.

[21] Fossati S., Cam J., Meyerson J., Mezhericher E., Romero I.A., Couraud P.O., Weksler B.B., Ghiso J., Rostagno A. // FASEB J. 2010. Vol. 24. N 1. P. 229-241.

[22] Schulte E.C., Fukumori A., Mollenhauer B., Hor $H$., Arzberger T., Perneczky R., Kurz A. // Eur. J. Hum Genet. 2015. Vol. 23. N 10. P. $1328-1333$.

[23] APP/ALZFORUM-URL:https://www.alzforum.org/mutations/ app (дата обращения 6.12.2019) 